\title{
Spatial presentation of cholesterol units on a DNA cube as a deter- minant of membrane protein-mimicking functions
}

\author{
Pongphak Chidchob ${ }^{1}$, Daniel Offenbartl-Stiegert ${ }^{2}$, Dillon McCarthy ${ }^{3}$, Xin Luo ${ }^{1}$, Jianing Li ${ }^{*}$, \\ Stefan Howorka ${ }^{{ }_{2}}$ and Hanadi F. Sleiman ${ }^{{ }_{1}}$ \\ ${ }^{1}$ Department of Chemistry, McGill University, Montreal, Quebec $\mathrm{H}_{3} \mathrm{~A}$ oB8, Canada \\ ${ }^{2}$ Department of Chemistry, Institute of Structural Molecular Biology, University College London, London $\mathrm{WC}_{1} \mathrm{H}$ \\ oAJ, United Kingdom \\ ${ }^{3}$ Department of Chemistry, The University of Vermont, Burlington, Vermont 05405, USA
}

\begin{abstract}
Cells use membrane proteins as gatekeepers to transport ions and molecules, catalyze reactions, relay signals, and interact with other cells. DNA nanostructures with lipidic anchors are promising as membrane protein mimics because of their high tuneability. However, the design features specifying DNA nanostructure's functions in lipid membranes are yet to be fully understood. Here, we show that altering patterns of cholesterol units on a cubic DNA scaffold dramatically changes its interaction mode with lipid membranes. This results in simple design rules that allow a single DNA nanostructure to reproduce multiple membrane protein functions: peripheral anchoring, nanopore behavior and conformational switching to reveal membrane-binding units. Strikingly, the DNA-cholesterol cubes constitute the first open-walled DNA nanopores, as only a quarter of their wall is made of DNA. This functional diversity can increase our fundamental understanding of membrane phenomena, and results in sensing, drug delivery and cell manipulation tools.
\end{abstract}

\section{Introduction}

DNA nanotechnology can achieve the structural organization of functional components into highly tunable patterns with exquisite precision. ${ }^{1-2}$ This field constitutes one of the most promising approaches for biomimicry, because of the selective and highly predictable nature of DNA hybridization, the sequence uniqueness of each component, and our excellent knowledge of DNA double helix structural parameters. Work in this field has greatly contributed to the development of artificial molecular systems that can carry out complex functions, and has significantly advanced fundamental understanding of biomolecular processes..$^{3-4}$

Membrane proteins are particularly relevant targets for biomimicry with DNA nanotechnology. ${ }^{5-7}$ Notably, much effort has involved the creation of membrane nanopores by learning from transmembrane protein designs. Several examples of membrane-spanning DNA nanostructures containing a protected internal channel were shown to regulate ion flux and small-molecule diffusion across the membrane. ${ }^{8-15}$ These structures feature a dense parallel alignment of DNA double helices into a barrel-like architecture. A second research focus has revolved around membrane shaping, remodeling and fusion. ${ }^{16-25}$ For instance, membrane deformation of lipid vesicles was demonstrated by oligomerizing rigid DNA monomers anchored on the membranes, ${ }^{20-23}$ and DNA templates were employed to shape and remodel liposomes with designer geometries. ${ }^{24-}$ ${ }^{25}$ Another exciting focus is membrane functionalization to control membrane's properties and interactions as well as to use membranes as platform for DNA self-assembly. ${ }^{26-36}$ As a recent example, cell clustering was programmed by higher-order assembly of DNA origami, imitating cell-adhesion molecules. ${ }^{37}$ These studies raise an important fundamental question: What are the general design rules that specify the behavior of DNA nanostructures with lipid bilayers, and can a combination of these structural variations be used to change functions of membrane protein mimics? This understanding, to our knowledge, is still at its very early stages. However, it will be necessary for the systematic development of DNA nanostructures as synthetic membrane protein mimics that overcome proteins' difficult bottom-up design.

Here, we set out to develop a modular DNA platform on which to organize cholesterol lipid anchors into different ${ }_{3} \mathrm{D}$ positions. The objective of this approach is to understand how structural factors govern the membrane function of DNA nanostructures. We find that factors such as number, orientation and rigidity of tethered cholesterol units on a wireframe DNA cube, are strong and predictable determinants of how a DNA structure interacts with a lipid bilayer. Cholesterol decoration on a single cube face leads to peripheral anchoring (Figure 1a), whereas cholesterol decoration at two cube faces causes membrane integration (Figure $1 b$ ), and nanopore function. ${ }^{8}{ }^{38}$ Importantly, the latter nanostructure is the first example of a DNA nanopore with open walls, in contrast to DNA-dense previous examples (Figure 1c). This striking finding raises fundamental questions on the mechanism of pore formation, 


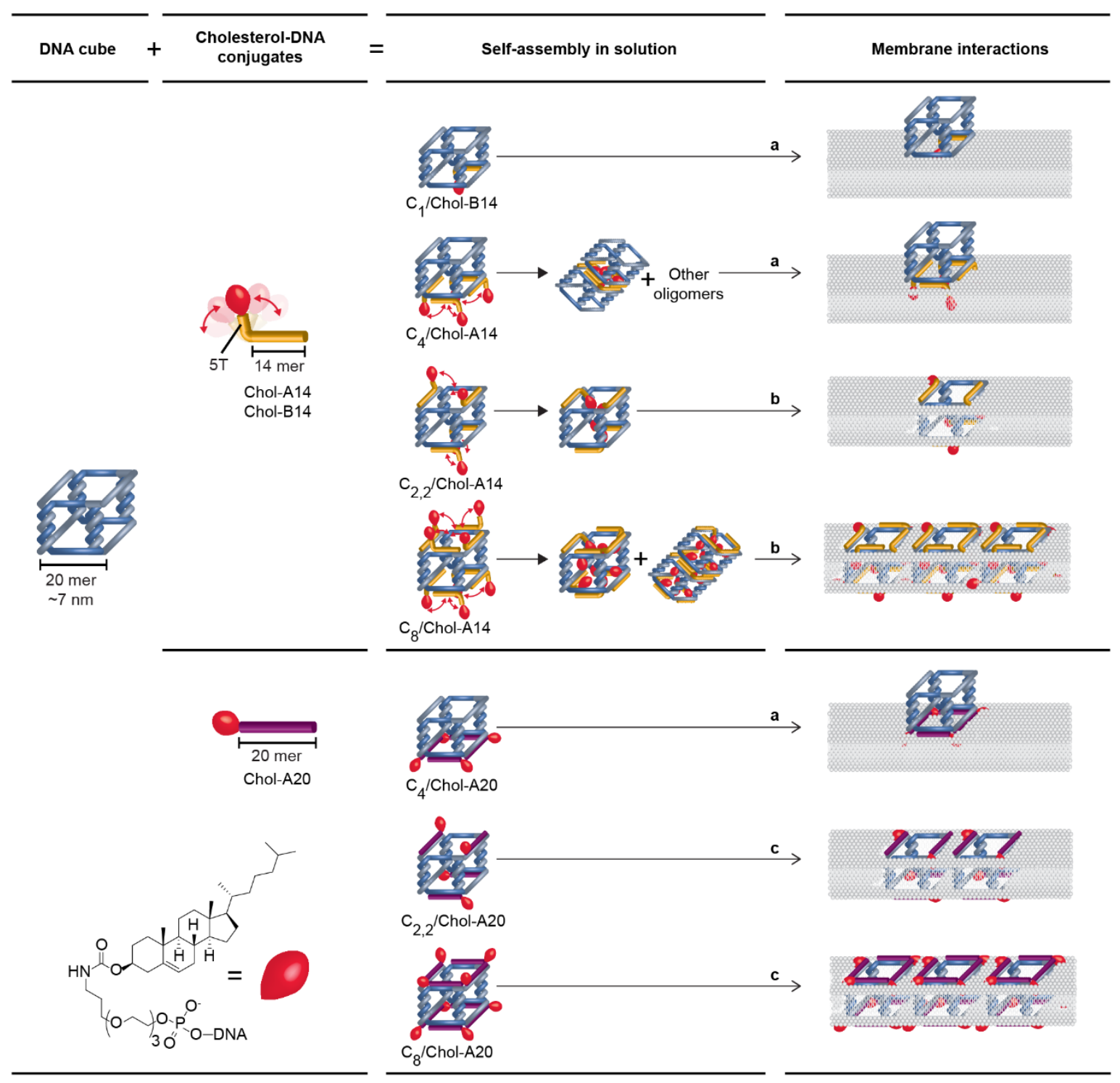

Figure 1. Design, assembly and membrane interactions of DNA/cholesterol nanostructures. DNA cubes serve as wireframe nanoscaffolds to provide $3 \mathrm{D}$ cholesterol presentations. The cholesterol units were organized on the cubes by selective hybridization between single-stranded DNA segments on the cubes (colored in blue and grey lines) and cholesterol-DNA conjugates of complementary sequences (colored in yellow and purple lines). Note that each solid line in the illustrations represents a single-stranded segment of the cubes and cholesterol-DNA conjugates. Both cube monomers (intramolecular assembly of cholesterol units) and oligomers (intermolecular assembly of cholesterol units) were assembly outcomes of the cubes decorated with multiple Chol-Ai4, while Chol-A2o functionalization generated cube monomers. Upon membrane binding, the nanostructures could either (a) anchor peripherally or (b) puncture the membrane, depending on their cholesterol orientation. (c) The poration activity was examined only in the cubes functionalized with Chol-A2o. The clustering and lateral mobility of our nanostructures can be further modulated by changing number, orientation and flexibility of cholesterol units.

which we preliminarily address using a molecular dynamics simulation. In addition, we tune the cubes' clustering and lateral mobility on lipid membranes by changing the configuration and flexibility of cholesterol units. ${ }^{39}$ Finally, one of the geometries allows the cholesterol units to 'hide' inside the cube and to then be exposed through a conformational switch upon membrane binding ${ }^{28}$, akin to the structural transition of Bax proteins and pathogenic poreforming toxins. ${ }^{4^{0-42}}$ Our design goes beyond previous studies by demonstrating that changing cholesterol positions can achieve membrane tethering and insertion within one platform structure. Thus we describe the first example of a single DNA nanostructure that predictably replicates a wide range of membrane protein interactions by simple chemical alteration.

\section{Results and Discussion}

Membrane interacting structures are built with DNA cubes and cholesterol-DNA strands. We selected a wireframe DNA cube as an addressable nanoscaffold (Figure 1, first column) to spatially organize lipid anchors and achieve predictable membrane-interacting modes. The cube is of $7 \mathrm{~nm}$ side length which is slightly larger than the lipid bilayer's thickness of $\sim 4^{-5} \mathrm{~nm}$. The DNA scaffold is composed of four 80 nucleotide (nt) DNA strands which hybridize with one another to yield a cube with four vertical duplexes and eight single- 

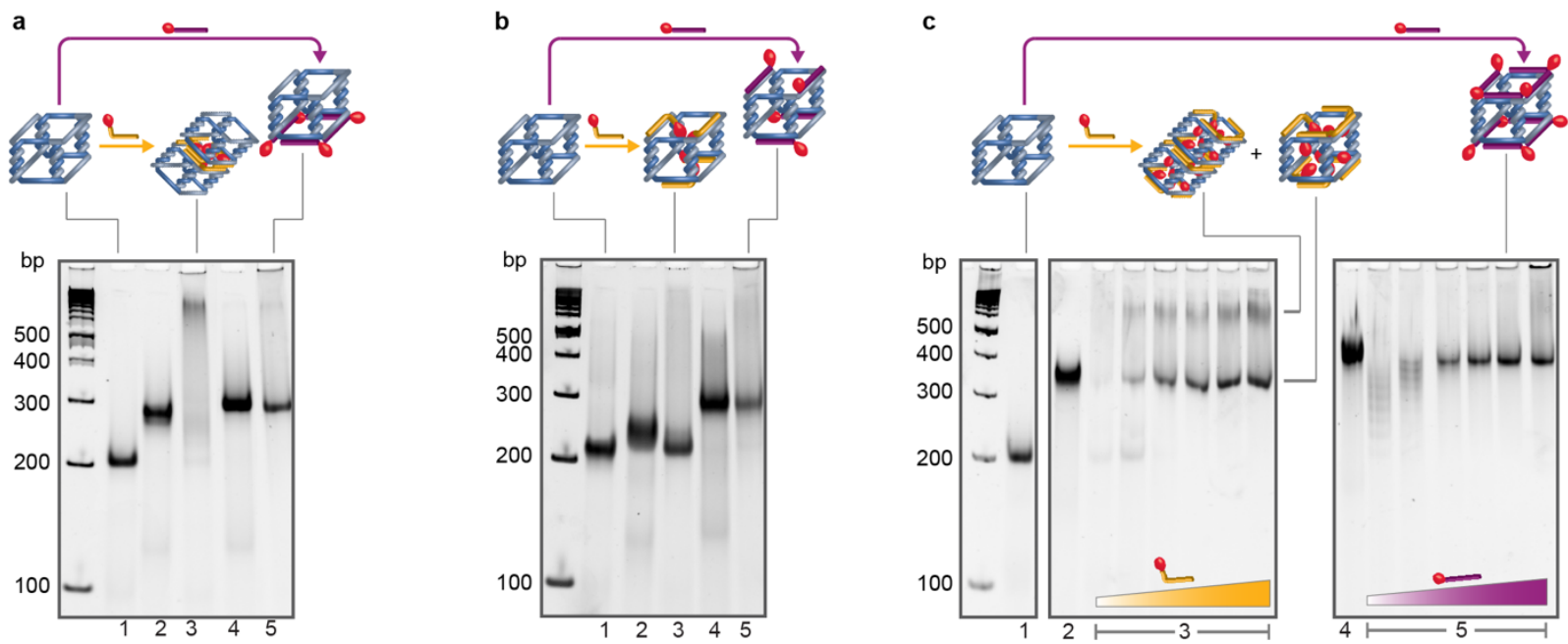

Figure 2. Assembly and characterization of DNA/cholesterol nanostructures. Non-denaturing polyacrylamide gel electrophoresis: lane 1 is DNA cube; lanes 2, 3, 4 and 5 are DNA cubes functionalized with A14, Chol-A14, A2o and Chol-A2o. (a) Intermolecular hydrophobic assembly between cholesterol units led to the aggregation of $\mathrm{C}_{4} /$ Chol-A14, whereas the main products of $\mathrm{C}_{4} / \mathrm{Chol}$ A2o were cube monomers. (b) Cube monomers were the main products for both $\mathrm{C}_{2,2} / \mathrm{Chol}_{-\mathrm{A}} 4$ and $\mathrm{C}_{2,2} / \mathrm{Chol}_{-} \mathrm{A2o}$. Only $\mathrm{C}_{2,2} / \mathrm{Chol}_{-}$ A14 showed the intramolecular assembly of cholesterol units inside the cube. (c) Cholesterol distribution on both faces of cube $\mathrm{C}_{8}$ yielded cube monomers and a minor population of cube dimers for $\mathrm{C}_{8} / \mathrm{Chol}-\mathrm{A}_{14}$, but only cube monomers for $\mathrm{C}_{8} / \mathrm{Chol}_{-} \mathrm{A} 2 \mathrm{O}$. Substoichiometric addition of Chol-A14 to cube $C_{8}$ showed 'all-or-none' cooperative binding behavior, which did not occur in the case of Chol-A2o. Concentrations of cholesterol-DNA conjugates are 4, 6, 8, 10, 12 and 16 equivalents with respect to cube $C_{8}$.

stranded segments at the top and bottom faces. ${ }^{43-44}$ These 20nt-long sites (Figure 1, first column) are of unique sequences, thereby allowing predictable hybridization of additional strands.

Cholesterol-modified DNA strand (Figure 1, second column) of matching complementary sequence were chosen to place the hydrophobic membrane anchors at up to eight sites of the DNA cube (Figure 1, third column). This rational design strategy is able to control the number, orientation, and flexibility of tethered cholesterol units.

To vary the number, cubes with one cholesterol $\left(C_{1}\right)$, four cholesterols $\left(C_{4}\right.$ and $\left.C_{2,2}\right)$, and eight cholesterols $\left(C_{8}\right)$ were selected (Figure 1 , third column). To vary the orientation, four-cholesterol cubes $\mathrm{C}_{4}$ and $\mathrm{C}_{2,2}$ carry the tags either on one cube face $\left(C_{4}\right)$, or each two cholesterol units on the two faces, arranged in a diagonal manner $\left(C_{2,2}\right)$. Based on their design, the $C_{1}$ and $C_{4}$ nanoscaffolds with cholesterol units on one cube face are expected to undergo peripheral binding to a lipid membrane, while cubes $C_{2,2}$ and $C_{8}$ can only anchor cholesterol units on both faces by integrating into the membrane.

As final design feature, the flexibility of tethered cholesterol units at the cube can be modulated. This is achieved by tuning the length of the cholesterol-DNA conjugate, and the length of the spacer between the strand's cubebinding segment and the cholesterol unit. The first strand, named 'A14' (or 'B14'), is complementary to only 14 bases of the cube's binding site. In addition, it carries a 5-thymidine (T) spacer between the binding region and the hydrophobic cholesterol unit (Figure 1, second panel). We previously showed that this partially complementary design gives flexibility to the cube, and allows for the interaction of alkyl chains inside the same cube (Figure 1, third panel). ${ }^{44-45}$ The other strand, termed 'A2o', is fully complementary to the 20-nt-long binding site. It features no thymidine spacer (Figure 1, second panel) and thus orients the cholesterol towards the cube's corners (Figure 1 , third panel). We reasoned that the resulting longer distance between cholesterol units as well as the increased nanostructure rigidity may reduce cholesterol-cholesterol interactions within these cubes.

Cholesterol tunes the assembly modes of DNA cubes by hydrophobic effect. We first investigated how the number, orientation, and flexibility of tethered cholesterol units controls the assembly behavior of our nanostructures in solution. The assembly outcome of different cube/cholesterol combinations was characterized by using non-denaturing polyacrylamide gel electrophoresis (Figure 2 and Supplementary Information, Figures 3-5) atomic force microscopy (Supplementary Information, Figure 7) and dynamic light scattering (Supplementary Information, Table $6)$. The analysis was first conducted for DNA cubes with flexible cholesterol units (Chol-A14).

The role of cholesterol number was examined by comparing cubes $C_{1}$ and $C_{4}$. The gel electrophoretic results confirmed successful formation of cholesterol-modified cube $\mathrm{C}_{1}$ as a single product (Supplementary Information, Figure 3). ${ }^{26}$ However, cube $C_{4}$ with four cholesterol units on one cube face yielded dimers, trimers and other oligomers, as evident by a smeary band in gel electrophoresis and oligomeric structures in AFM (Figure 2a, lane 3; Supplementary Information, Figure 4). Cholesterol number hence controls assembly into cube oligomers, most likely by hydrophobic interactions between the cholesterol units on the cube faces (Figure 1, top panel).

To probe the role of cholesterol orientation, we compared the assembly modes of cubes $C_{4}$ and $C_{2,2}$ that contain four cholesterol units at different positions. While cube $C_{4}$ 
formed inter-particle oligomers, the alternative cube $C_{2,2}$ migrated as a single band of increased mobility (Figure $2 \mathrm{~b}$, lane 3), compared to a non-cholesterol control (Figure $2 \mathrm{~b}$, lane 2). We previously showed that the folding of alkyl chains within the cube results in structure compaction and faster gel mobility. ${ }^{44-45}$ Thus, the fast migration for cube $C_{2,2}$ most likely represents a compact cube structure formed by the intramolecular attraction of cholesterol units inside the cube cavity.

The effect of number and orientation on intra- and intermolecular interaction was also observed for fully cholesterol-modified cube $C_{8}$. The cube assembly resulted in $C_{8}$ monomers that migrated faster than the equivalent structure with no cholesterol (Figure 2c, lane 2), consistent with intramolecular folding of the cholesterols into the cube interior. In addition, a minor population of cube dimers formed (Figure 2c, lane 3) which are most likely associated by hydrophobic effect. Strikingly, substoichiometric concentrations of cholesterol-DNA conjugate vs DNA cube revealed a 'all-or-none' binding behavior. As such, cubes formed with all eight or no cholesterol tags but not with intermediate cholesterol numbers (Figure 2c, lane 3). The data strongly suggests a cooperative mechanism for cube assembly mediated by the intramolecular hydrophobic effect, ${ }^{45}$ which was also found for cube $C_{2,2}$ (Supplementary Information, Figure 5). Interestingly, when cube $\mathrm{C}_{2,2}$ 's rigidity was increased by hybridizing its non-cholesterol binding sites with fully-complementary DNA strands ('B2O'), Chol-A14 binding to this cube became non-cooperative (Supporting Information, Figure 8). This demonstrates that a degree of cube flexibility is required for intramolecular folding of cholesterol units.

Finally, we probed the influence of flexibility of the cholesterol-tether as well as the scaffold, by replacing the above used Chol-A14 conjugate with Chol-A2o. In line with the less flexible Chol-A2o, no inter- or intramolecular association by cholesterol units was found for cubes $\mathrm{C}_{8}, \mathrm{C}_{4}$, and $C_{2,2}$ (Figure 2, lanes 5); normally migrating monomers were the major product (Figure $2 \mathrm{c}$, lane 5 for $\mathrm{C}_{8} / \mathrm{Chol}-\mathrm{A}_{20}$; see Supplementary Information, Figures 4-5 for other cubes). In contrast to its flexible counterpart, cube $C_{8}$ showed no cooperative behavior at substoichiometric concentrations of Chol-A2o, consistent with the lack of intramolecular interactions between the cholesterol units. It is remarkable that Chol-Azo cube's increased rigidity and longer inter-cholesterol distance can together overcome the inherent clustering tendency of cholesterols observed for flexible Chol-A14 cubes. ${ }^{46}$ Additional experiments where a $5 \mathrm{~T}$ spacer was added to Chol-A2o showed non-cooperative binding to all cubes, generating cube monomers for $C_{4}$ and $C_{2,2}$ but big aggregates as major products for $C_{8}$. This further supports that inter-cholesterol distance is important for altering intramolecular cholesterol interactions (Supplementary Information, Figure 6).

Thus, we have generated a library of DNA nanostructures with different cholesterol configurations of predictable behavior. Increasing the number of cholesterol units on a DNA cube leads to intermolecular clustering via hydrophobic cholesterol interactions. Changing cholesterol orientation by placing them on two rather than one cube face causes intramolecular cholesterol association within the cube. However, this requires flexibility in the cube and longer spacers. Conversely, increasing the rigidity of the scaffold greatly attenuates cholesterol interactions within and between DNA cubes.

Cholesterol configuration defines lateral membrane mobility and bilayer clustering of DNA nanostructures. To elucidate how cholesterol determines the cube's interactions with the membrane, we monitored their binding onto lipid vesicles with confocal laser scanning microscopy to determine the distribution of cubes on the membrane (Figure 3), and fluorescence recovery after photobleaching (FRAP) to quantify how fast cubes diffuse on the membrane surface. The nanostructures were labelled by attaching a $\mathrm{Cy}_{3}$ fluorophore at the 5' terminus of one of their DNA component strands. In these experiments, giant unilamellar vesicles (GUVs) composed of DOPC lipids were used as the lipid model system.

Microscopic characterization of individual GUVs first established that cholesterol tagging of DNA cubes was required for membrane binding. Cube with cholesterol units (via Chol-A2o) caused homogeneously bright, fluorescent rings of the vesicles while cubes without cholesterol yielded little fluorescence (Supplementary Information, Figure 9). As additional reference, the FRAP recovery halftime of cube $C_{1}$ on membranes was similar to a control strand with a cholesterol tether and the $\mathrm{Cy} 3$ fluorophore (Figure $3 \mathrm{e}$ ).

We next examined how the number of cholesterol units influences membrane interaction by comparing cube $C_{1}$ with $C_{4}$ and $C_{8}$. Previous gel analysis had established that multiple cholesterols led to a solution-based intermolecular clustering for cube $\mathrm{C}_{4}$. Hence, the key question was whether cholesterol-modified cubes would retain this behavior once exposed to membranes, or respond to the new hydrophobic bilayer environment by dynamically rearranging their cholesterol units. The microscopy analysis suggests that cholesterol units rearranged, because cube $C_{4}$ was homogeneously distributed on the membrane similar to non-clustering cube $C_{1}$ (Figure 3a; Supplementary Information, Figure 10). The homogenous distribution implies that the solution clusters of $\mathrm{C}_{4}$ dissociate into monomers on the vesicle surface and thereby expose their cholesterolface for membrane tethering (Figure 3a). In comparison, cube $\mathrm{C}_{8}$ led to a heterogeneous distribution with multiple high fluorescence patches on the vesicles, implying significant clustering of this nanostructure within the lipid membrane (Figure 3c). This clustering process could occur before or after binding to the membrane, and the monomeric cubes and lower-order oligomers of cube $C_{8}$ are the most likely structures to efficiently span the membrane. On the other hand, we observed extremely low binding efficiency when there is a significant aggregation degree of the nanostructure (Supplementary Information, Figure 11).

To further elucidate how the number of cholesterol units influences membrane interaction, FRAP was applied to determine the extent of mobile cubes and their FRAP recovery half-time. In line with the observed homogeneous distribution, cube $\mathrm{C}_{4}$ had a very high mobile fraction of $>90 \%$ 


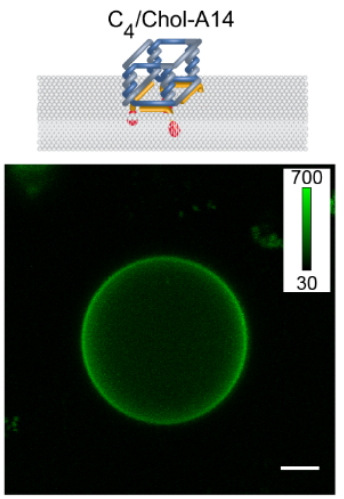

d

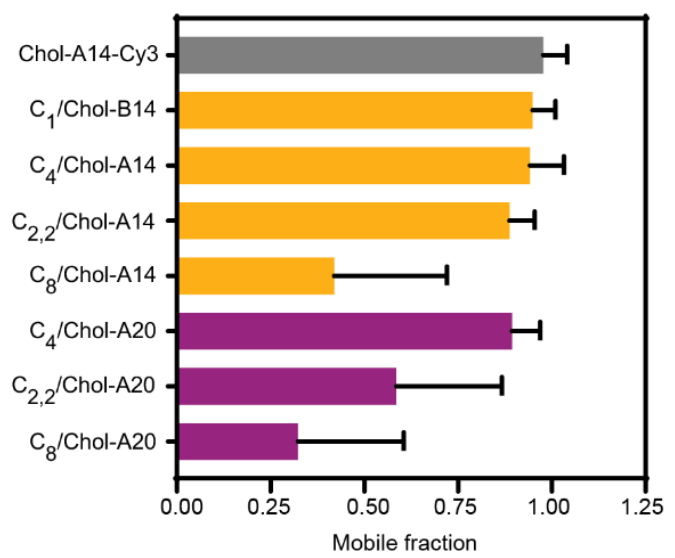

b

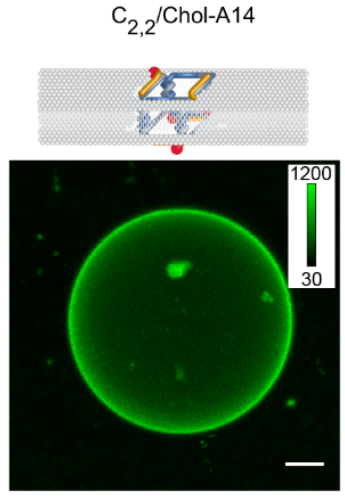

c $\quad \mathrm{C}_{8} /$ Chol-A14

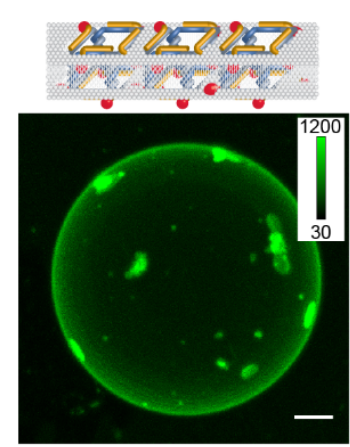

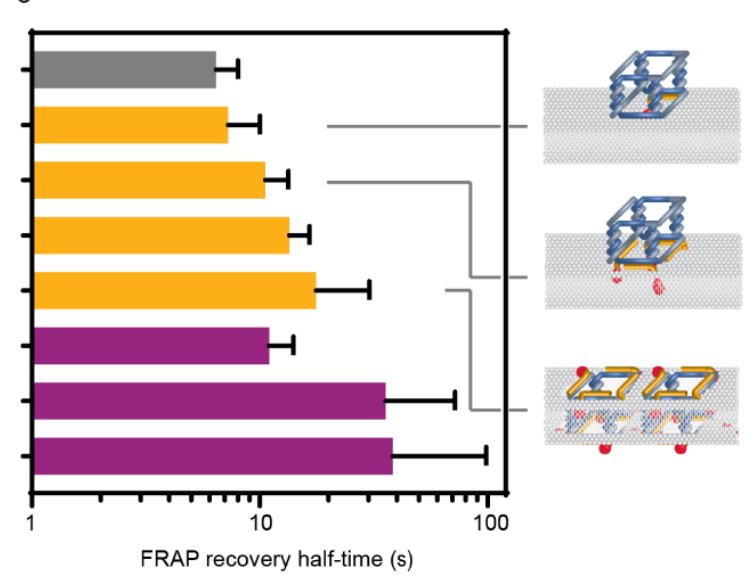

Figure 3. Binding of DNA/cholesterol nanostructures on DOPC giant lipid vesicles. Reconstructions of z-stacked confocal images

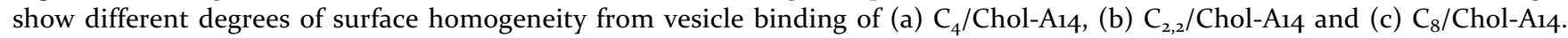
Additional confocal images can be found in Supplementary Information, Figure 10. FRAP assays were then performed for quantitative measurements. Color scale bar indicates $\mathrm{Cy}_{3}$ fluorescence intensity of DNA/cholesterol nanostructures. Length scale bar is $5 \mu \mathrm{m}$. (d) There was an inverse correlation between lateral membrane mobility and cholesterol number, consistent with stronger binding from multiple cholesterol units. Substitution of Chol-A14 with Chol-A2o can lower the mobility of cubes $\mathrm{C}_{2,2}$ and $\mathrm{C}_{8}$ by reducing the extent of intramolecular assembly of cholesterol units inside the cubes. (e) Eight cholesterol units caused a significant decrease in mobile fraction, indicative of clustering degree. Cholesterol sequestering inside DNA cubes by intramolecular assembly could attenuate the clustering, especially in cube $C_{2,2}$.

similar to single-cholesterol tethered $C_{1}$ (Figure 3e). However, FRAP recovery half-time for cube $C_{4}$ was significantly higher than for cube $C_{1}$, (Figure $3 e$ ) mostly likely as the tethering of the cubes via multiple as opposed to one cholesterol anchor slowed down lateral movement at the membrane surface. ${ }^{47}$ In comparison, cube $C_{8}$ showed a significantly reduced mobile fraction (Figure ze) consistent with clustering at the membrane caused by the hydrophobic effect. Furthermore, cube $C_{8}$ diffused significantly slower than cube $\mathrm{C}_{4}$, which may also likely reflect a membrane-spanning rather than the $C_{4}$ cube's membrane-tethered status, as diffusion coefficients of integral membrane proteins are about half as fast as peripheral proteins ${ }^{48-49}$ (see below for additional evidence of membrane spanning). Therefore, increasing the number of cholesterol units decreases the surface mobility and increases membrane clustering of DNA nanostructures. ${ }^{47}$ However, these effects could be weakened by reducing the nanostructure's concentration in comparison to GUVs (Supplementary Information, Figures 14-15). We noted that this reduction will be highly beneficial in applications where the consistency in our nanostructure's behavior throughout the vesicle surface is required.

The effect of cholesterol orientation on lipid membrane interactions was examined by comparing cubes $C_{4}$ and $C_{2,2}$. The previously suggested membrane-spanning state for cube $C_{2,2}$ was supported by the less homogeneous fluorescence distribution (Figure $3 \mathrm{~b}$ ), the lower mobile fraction, and the slower mobility when compared to cube $C_{4}$ (Figure $3 \mathrm{~d}, \mathrm{e})$. We note that the clustering behavior as indicated by low mobile fraction could be strongly driven by the lateral interactions between the nanostructures. Due to the presence of cholesterol units on both faces of cubes $C_{2,2}$ and $C_{8}$, these nanostructures could have a better interfacial contact with one another, leading to enhanced cube/cube interactions and thus clustering.

Finally, the effect of scaffold rigidity on lipid membrane interactions was examined. We showed earlier that flexible DNA cubes with cholesterol units on both faces $\left(\mathrm{C}_{2,2} / \mathrm{Chol}-\right.$

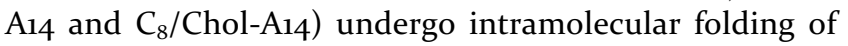
their cholesterol units into the inside of the cube, with corresponding assembly cooperativity (Figure 1 top). 


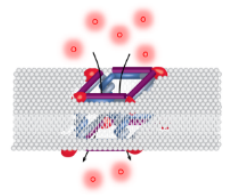

$0 \mathrm{~s}$
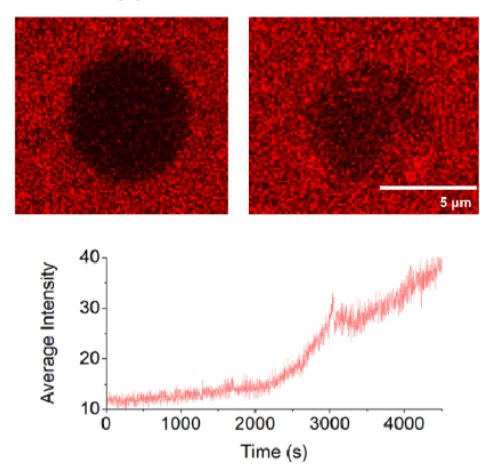

b

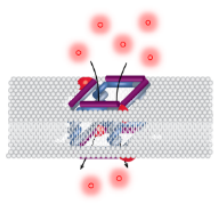

$0 \mathrm{~s}$
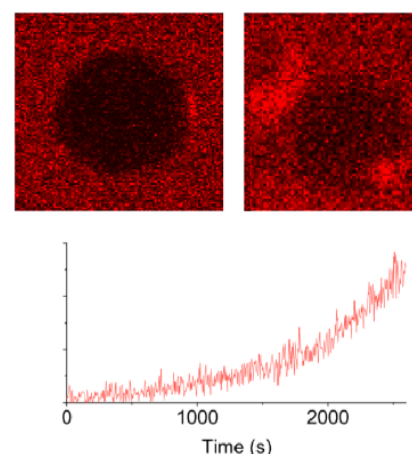

$2600 \mathrm{~s}$
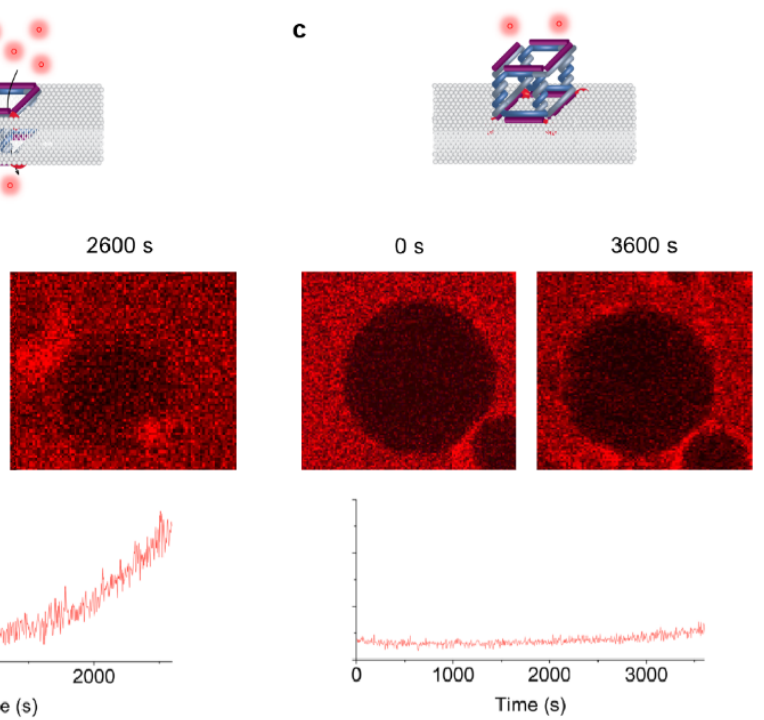

Figure 4. The dye influx assay with $\mathrm{C}_{8} /$ Chol-A2o, $\mathrm{C}_{2,2} / \mathrm{Chol-A20/B20}$ and $\mathrm{C}_{4} / \mathrm{Chol}-\mathrm{A}_{20} / \mathrm{B} 20$ using POPC GUVs exposed to fluorophore ATTO 633. The cubes with cholesterol units on two opposing faces (cubes $C_{8}$ and $C_{2,2}$ ) cause fluorophore influx and likely span the membrane. The cubes with cholesterol units on only one face (cube $C_{4}$ ) do not show significant influx and are assumed to float on the membrane.

Thus, membrane binding would require conformational switching of the cholesterol units from the internal cube space to the exterior. The bright fluorescent rings observed upon binding flexible cubes $C_{2,2}$ and $C_{8}$ indeed suggest that they undergo conformational switching via an 'in-thenout' display of hidden cholesterol units upon membrane exposure. This 'in-then-out' mechanism was previously incorporated into DNA origami by List el al. ${ }^{28}$ These structural dynamics share a similarity to the membrane-triggered exposure of hydrophobic domains in several monomers of bacterial pore-forming toxins. ${ }^{40}$ On the other hand, the more rigid cubes $\mathrm{C}_{2,2} /$ Chol-A2o and $\mathrm{C}_{8} / \mathrm{Chol}-\mathrm{A} 2 \mathrm{O}$ do not display cholesterol self-interactions, thus their cholesterol units may be more exposed and available (Figure 1 bottom). This was manifested both in their reduced membrane mobility and increased clustering properties (Figures 3 d-e), as compared to flexible cubes. Additional experiments to prevent the conformational switching of $\mathrm{C}_{2,2} / \mathrm{Chol}_{-\mathrm{A}} 4 \mathrm{by}$ improving its rigidity also led to increased membrane interactions (Supplementary Information, Figure 17)

In contrast to the membrane spanning cubes $C_{2,2}$ and $C_{8}$, cube $C_{4}$ is not expected to display drastically different membrane behavior between its flexible (A14) and rigid (A2o) forms. This is because the flexible $\mathrm{C}_{4} / \mathrm{Chol}$-A14 does not show significant cholesterol intramolecular interactions (Supplementary Information, Figure 4), thus these units are exposed and available in both flexible and rigid forms. Indeed, the extent of mobile fraction and the recovery half-time were the same for $\mathrm{C}_{4} / \mathrm{Chol}-\mathrm{A} 2 \mathrm{O}$ and $\mathrm{C}_{4} / \mathrm{Chol}$ A14 (Figure 3d, e).

We can conclude that our nanostructures efficiently bind to lipid vesicles through cholesterol-lipid interactions. Their membrane-binding characteristics can be tuned by changing the number, orientation and flexibility of their cholesterol units. Increasing the number of cholesterol units, or distributing these units on both faces of the cube lead to slower membrane mobility and higher clustering degree. It is of note that the membrane-binding density could potentially affect the membrane diffusion of DNA nanostructures. ${ }^{20,47}$ Although this is not a focus of our study, additional experiments will be beneficial to probe and further understand this effect. Finally, unique to our design, placing cholesterol units on both faces of a flexible cube results in intramolecular interactions between these units, leading to faster membrane mobility than rigid cubes.

Decorating cholesterol units on both faces of DNA cubes is key for membrane spanning. To confirm that hollow cubes $C_{4}$ and $C_{2,2}$ can span a membrane bilayer, a dye influx assay was carried out (Figure 4). In the assay, DNA cubes functionalized with Chol-A2o were added to lipid vesicles that were immersed in fluorophore-containing solution. The cubes able to puncture the membrane were expected to facilitate dye influx into vesicles. By comparison, cube $\mathrm{C}_{4}$ assumed to float on the membrane without puncturing should not lead to influx. The cubes also featured non-functionalized $\mathrm{B} 2 \mathrm{O}$ to fill the remaining single-stranded segments on $\mathrm{C}_{4} /$ Chol-A2o and $\mathrm{C}_{2,2} /$ Chol-A2o to further increase their rigidity.

The results of an influx assay with POPC GUVs and a small fluorophore dye ATTO 633 of around $1 \mathrm{~nm}$ size are summarized in Figure 4. The fluorescence intensity within the vesicles was tracked using confocal microscopy and plotted as a function of time. As shown in Figures 4a and $4 \mathrm{~b}$, the suggested membrane-spanning $\mathrm{C}_{8} /$ Chol-A2o and $\mathrm{C}_{2,2} / \mathrm{Chol}-\mathrm{A2O} / \mathrm{B2O}$ caused an increase in fluorescence inside the vesicle. In further agreement, $\mathrm{C}_{4} / \mathrm{Chol}-\mathrm{A} 2 \mathrm{O} / \mathrm{B} 2 \mathrm{O}$ thought to float on the membrane did not lead to influx (Figure $4 \mathrm{C}$ ). To further confirm that $\mathrm{C}_{8} /$ Chol-A2o and $\mathrm{C}_{2,2} / \mathrm{Chol}-\mathrm{A20} / \mathrm{B} 2 \mathrm{O}$ puncture the membrane, the size-dependent permeation was examined with FITC-Dextran dye with a higher molecular weight of $500 \mathrm{kD}$ and a diameter of $>15 \mathrm{~nm}$. The probe is too large to fit through the lumen 


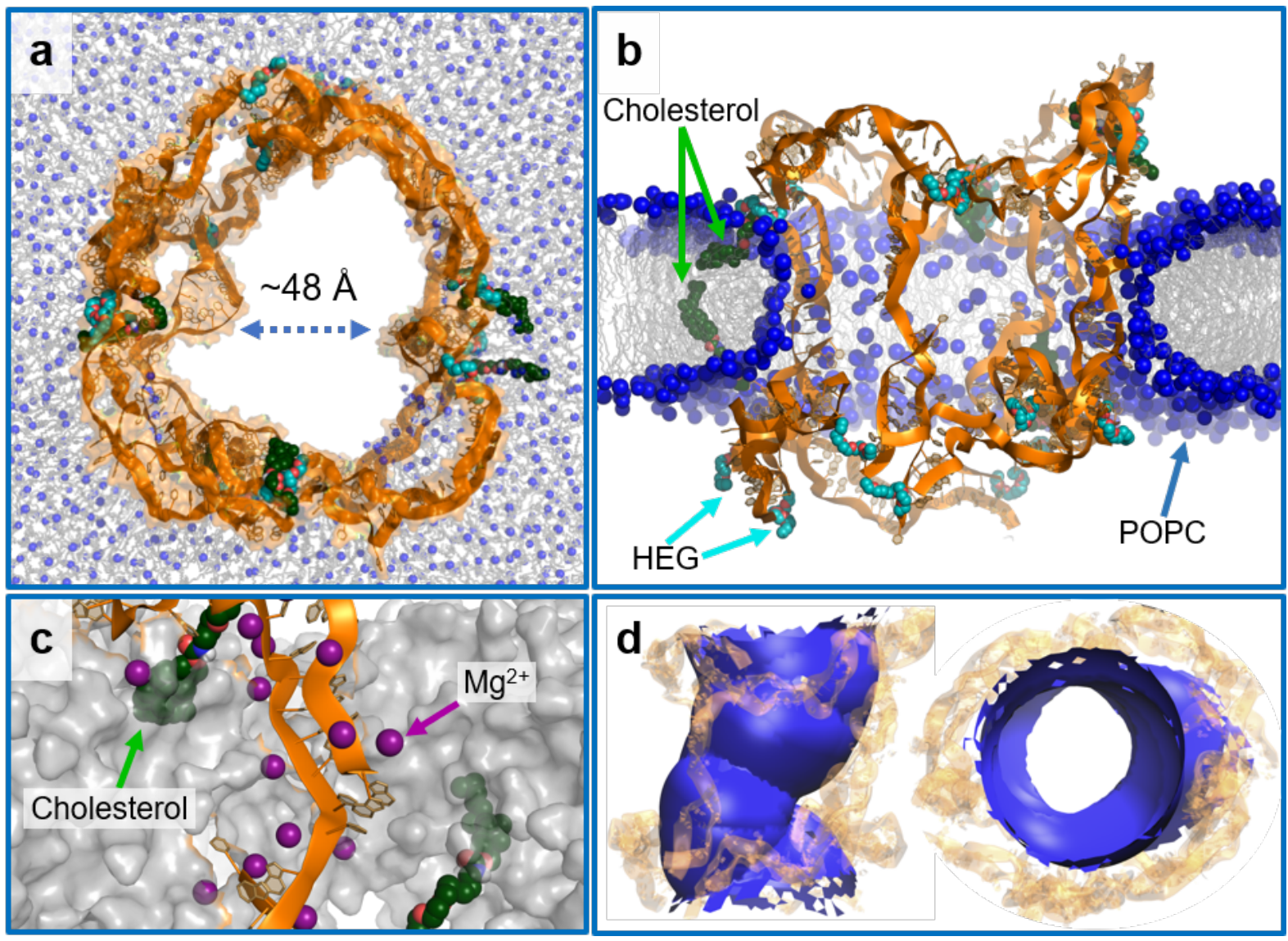

Figure 5. Snapshots of $C_{8} /$ Chol-A2o (orange ribbons) in MD simulations. (a) Top down view of the cube with cholesterol units pre-inserted in the POPC bilayer (snapshot at $50 \mathrm{~ns}$ ). (b) Sideview of the cube without pre-inserted cholesterol units (snapshot at $50 \mathrm{~ns}$ ). The $\mathrm{P}$ and $\mathrm{N}$ atoms (blue spheres) are shown to highlight the membrane curvature with direct lipid-DNA contacts. (c) The DNA/POPC $/ \mathrm{Mg}^{2+}$ interface. (d) Illustrations of the nanopore from the side (left) and the top (right). DNA cube shown as transparent orange ribbons.

of DNA/cholesterol nanostructures but can pass across induced lesions within the GUV membranes.. "Fluorescence analysis revealed that neither $\mathrm{C}_{8} / \mathrm{Chol}-\mathrm{A} 2 \mathrm{O}$ nor $\mathrm{C}_{2,2} / \mathrm{Chol}$ A2o/B2o facilitated influx of the large dye (Supplementary Information, Figure 19), thereby corroborating the cubes' bilayer-spanning nature rather than any non-specific membrane destabilization. ${ }^{50}$ As another control, the flexible $\mathrm{C}_{8} / \mathrm{Chol}^{-\mathrm{A}} 14$ also allowed the ATTO 633 influx into the vesicle, further confirming that this nanostructure can span the membrane (Supplementary Information, Figure 19). Additionally, the membrane-spanning behavior of our DNA nanostructures were also supported by their increased resistance towards enzymatic digestion ${ }^{26,50}$ (Supplementary Information, Figure 22) and lower height on supported lipid bilayers measured by AFM (Supplementary Information, Figure 25).

Molecular dynamics simulations reveal the formation of a stable DNA nanopore. As molecular dynamics simulations have been applied to DNA nanotechnology, $8,15,51$ we used the simulation to understand how the membrane remodels to accommodate the DNA cube and to visualize the channel formation as well as its dimensions. As such, all-atom molecular dynamics (MD) simulations on cube $C_{8}$ with rigid cholesterol units (Chol-A2o) were performed to study the detailed interactions between the DNA cube, the cholesterol decorations, and the POPC bilayer. To assess the pore stability, two models of up to one million atoms were setup and simulated for $\sim 50$ ns. The first starting model was constructed with the cholesterol decorations inserted into the bilayer. This simulation suggests that while the structure of the cube in the bilayer is highly dynamic, its membrane-spanning behavior is nevertheless stable for at least $50 \mathrm{~ns}$, resulting in the formation of a channel with a pore radius of $\sim 24 \AA$ (Figure $5 \mathrm{a}$ and $5 \mathrm{~d}$ ). Also, we observe rapid remodeling of the POPC bilayer as the charged headgroups fold over to maximize their contact with the DNA backbone, which eventually leads to the formation of a charged POPC surface which surrounds the cube (Figure 5b). A closer examination of the DNA/POPC interface reveals the presence of magnesium ions surrounding the DNA backbone, especially midway between the POPC headgroups and DNA backbone (Figure $5 \mathrm{c}$ ). Magnesium ions hence play a role in increasing the overall nanopore stability.

Moreover, all cholesterol decorations remain embedded within the POPC bilayer for the duration of the simulation. This observation is consistent with our hypothesis that the hydrophobic nature of cholesterol is a driving force for pore formation. To further test this hypothesis, a second construct without initial cholesterol insertion was simulated for $\sim 67$ ns. Fast embedding of the cholesterol in membranes within $\sim 20$ ns was observed in addition to 
rapid membrane remodeling (Supplementary Information Video). $\mathrm{C}_{8} /$ Chol-A2o, while relatively rigid, has hexaethylene glycol (HEG) linkers on its 8 corners, which likely provide some flexibility for its deformation inside the membrane. While both simulations show direct evidence of a stable nanopore, the simulation of the second construct ultimately yielded a more deformed DNA cube. This simulation indicates that cholesterol might also be important for pore stability, in conjunction with the interactions between the cube, the POPC bilayer, and the magnesium ions.

\section{Conclusion}

The ability to use a single structural platform to dial into a range of multiple membrane functions is the conceptual advance in our study. Cholesterol orientation and scaffold flexibility are able to direct assembly modes of cholesterol units tethered on the cubes, thereby controlling their membrane-interacting behavior. Single-face cube decoration with cholesterol units leads to peripheral membrane anchoring, in which the surface mobility can be modulated by cholesterol number. On the other hand, decorating both cube faces with cholesterol units leads to membrane insertion. ${ }^{8,} 3^{8}$ The DNA nanostructures are then able to puncture the membrane, allowing the passage of small molecules. Increasing the number of cholesterol units decreases their surface mobility and increases their clustering degree. Finally, nanostructures that have a flexible cholesterol configuration can undergo structural 'in-then-out' switching to expose their hidden cholesterol units upon membrane binding. ${ }^{28}$ We thus present a modular DNA nanostructure of cube shape that can be predictably designed to exhibit various modes of membrane interactions.

It is important to note that typical DNA-based channels are 'barrel-like' architectures enclosed by DNA-dense walls, ${ }^{52}$ whereas our wireframe nanostructures have large gaps on their walls. Thus, our design is the most DNAminimal channel reported to date. As such, it is possible that that lipid molecules reside in the pore created by our nanostructure. If this were the case, then these lipids might have a different packing behavior compared to those surrounding the pore, and this membrane disruption could influence membrane transport. Furthermore, a top and a bottom layer of cholesterol units on both faces of the structure are important for membrane puncturing. This leads to an interesting question regarding the mechanism of membrane insertion: when the first cholesterol layer inserts into the lipid bilayer, how is the second cholesterol layer 'sensed' by the membrane to allow full integration? These cholesterol layers are separated by approximately two full turns of polyanionic DNA helices. In addition, our DNA cube contains flexible hexaethylene glycol linkers at each corner. This flexibility might be important to increase nanostructure's flexibility, and it may help molecular recognition and membrane insertion. Further molecular modeling analysis is necessary to better understand the poration mechanism of our nanostructures.

A direct application of the approach presented in this work is on membrane interfaces. The advantage of our design is that the open spaces created by the DNA scaffold can be easily decorated with different structural parts to add new functions depending on the desired use. For example, one could add peptide or proteins to chemically define the pore wall structure and thus its function such as the interaction with transported cargo. It is also conceivable to add receptors or enzymes into the pore wall for sensing or biocatalytic applications. A recent study by Bayley et $a l$. has added to a non-membrane spanning DNA ring peptide that span the lipid bilayer..$^{53}$ Our design adds more tunability as different parts of the pore can be decorated with different functional units. This modification will be useful for membrane engineering. Moreover, several biological functions rely on the hierarchical organization through surface clustering of membrane proteins. ${ }^{54}$ The clustering of our nanostructures can be used to probe and influence this effect on lipid membranes. Excitingly, membrane-spanning behavior of our nanostructures can be harnessed as synthetic channels for membrane transport. Reconfigurable DNA channels are highly possible as different cage geometries can be easily generated such that the width of the lumen can be tuned, thus increasing the potential applications of the structures. It is also viable to incorporate stimuli-responsive pore opening/closing mechanisms as the gatekeeper, which is useful for biophysical tools, cellular imaging and drug delivery. ${ }^{9}$ Apart from the cholesterol modification, different lipid anchors could be attached on different positions of the cubes, opening an exciting avenue to further tune membrane-binding affinity and lipid-phase selectivity of DNA nanostructures. ${ }^{5-6}$ Therefore, we anticipate that our design approach will be highly useful to a wide range of applications in biomedical nanotechnology and material sciences.

\section{ASSOCIATED CONTENT}

Supporting Information. This material is available free of charge via the Internet at http://pubs.acs.org.

DNA cage design and assembly, lipid vesicle preparation, membrane-binding study, dye-influx assay and molecular dynamic simulations.

\section{AUTHOR INFORMATION}

\section{Corresponding Author}

*hanadi.sleiman@mcgill.ca

*s.howorka@ucl.ac.uk

*jianing.li@uvm.edu

Notes

The authors declare no competing financial interest.

\section{ACKNOWLEDGMENT}

H.F.S. and P.C. would like to thank NSERC, CFI, CSACS and FRQNT and the NSERC CREATE training program in Bionanomachines for funding. H.F.S. is a Cottrell Scholar of the Research Corporation. H.F.S. and P.C. also thank the Advanced BioImaging Facility (McGill University) for their help with CLSM and FRAP experiments. S.H. is supported by the EPSRC (EP/Noog282/1), the BBSRC (BB/Mo25373/1, BB/No17331/1), and the Leverhulme Trust (RPG-2017-015). D.M. and J.L. thank Nvidia for the GPU grant, and the computer resources provided by the Vermont Advanced Computing Core. 


\section{REFERENCES}

1. Aldaye, F. A.; Palmer, A. L.; Sleiman, H. F., Assembling Materials with DNA as the Guide, Science 20o8, 321, 1795-9.

2. Seeman, N. C.; Sleiman, H. F., DNA Nanotechnology, Nat. Rev. Mater. 2017, 3, 17068.

3. Zhang, F.; Nangreave, J.; Liu, Y.; Yan, H., Structural DNA Nanotechnology: State of the Art and Future Perspective, $J$. Am. Chem. Soc. 2014, 136, 11198-11211.

4. Yang, Y. R.; Liu, Y.; Yan, H., DNA Nanostructures as Programmable Biomolecular Scaffolds, Bioconjugate Chem. 2015, 26, 1381-95.

5. Czogalla, A.; Franquelim, H. G.; Schwille, P., DNA Nanostructures on Membranes as Tools for Synthetic Biology, Biophys. J. 2016, 110, 1698-1707.

6. $\quad$ Langecker, M.; Arnaut, V.; List, J.; Simmel, F. C., DNA Nanostructures Interacting with Lipid Bilayer Membranes, Acc. Chem. Res. 2014, 47, 1807-15.

7. Howorka, S., Changing of the Guard, Science 2016, 352, 890-1.

8. Gopfrich, K.; Li, C. Y.; Mames, I.; Bhamidimarri, S. P.; Ricci, M.; Yoo, J.; Mames, A.; Ohmann, A.; Winterhalter, M.; Stulz, E.; Aksimentiev, A.; Keyser, U. F., Ion Channels Made from a Single Membrane-Spanning DNA Duplex, Nano Lett. 2o16, 16 , 4665-9.

9. Burns, J. R.; Seifert, A.; Fertig, N.; Howorka, S., A Biomimetic DNA-Based Channel for the Ligand-Controlled Transport of Charged Molecular Cargo across a Biological Membrane, Nat. Nanotechnol. 2016, 11, 152-156.

10. Langecker, M.; Arnaut, V.; Martin, T. G.; List, J.; Renner, S.; Mayer, M.; Dietz, H.; Simmel, F. C., Synthetic Lipid Membrane Channels Formed by Designed DNA Nanostructures, Science 2012, 338, 932-6.

11. Krishnan, S.; Ziegler, D.; Arnaut, V.; Martin, T. G.; Kapsner, K.; Henneberg, K.; Bausch, A. R.; Dietz, H.; Simmel, F. C., Molecular Transport through Large-Diameter DNA Nanopores, Nat. Commun. 2016, 7, 12787.

12. Burns, J. R.; Gopfrich, K.; Wood, J. W.; Thacker, V. V.; Stulz, E.; Keyser, U. F.; Howorka, S., Lipid-Bilayer-Spanning DNA Nanopores with a Bifunctional Porphyrin Anchor, Angew. Chem. Int. Ed. 2013, 52, 12069-72.

13. Burns, J. R.; Stulz, E.; Howorka, S., Self-Assembled DNA Nanopores That Span Lipid Bilayers, Nano Lett. 2013, 13, 23512356.

14. Seifert, A.; Gopfrich, K.; Burns, J. R.; Fertig, N.; Keyser, U. F.; Howorka, S., Bilayer-Spanning DNA Nanopores with Voltage-Switching between Open and Closed State, ACS Nano 2015, 9, 1117-1126.

15. Gopfrich, K.; Li, C. Y.; Ricci, M.; Bhamidimarri, S. P.; Yoo, J.; Gyenes, B.; Ohmann, A.; Winterhalter, M.; Aksimentiev, A.; Keyser, U. F., Large-Conductance Transmembrane Porin Made from DNA Origami, ACS Nano 2016, 10, 8207-14.

16. Stengel, G.; Zahn, R.; Höök, F., DNA-Induced Programmable Fusion of Phospholipid Vesicles, J. Am. Chem. Soc. 2007, 129, 9584-9585.

17. Versluis, F.; Voskuhl, J.; van Kolck, B.; Zope, H.; Bremmer, M.; Albregtse, T.; Kros, A., In Situ Modification of Plain Liposomes with Lipidated Coiled Coil Forming Peptides Induces Membrane Fusion, J. Am. Chem. Soc. 2013, 135, 8057-8062.

18. Chan, Y. H.; van Lengerich, B.; Boxer, S. G., Effects of Linker Sequences on Vesicle Fusion Mediated by Lipid-Anchored DNA Oligonucleotides, Proc. Natl. Acad. Sci. U.S.A. 2009, 106, 979-84

19. $\mathrm{Xu}, \mathrm{W} . ;$ Pincet, F.; Shih, W.; Rothman, J. E., A Programmable DNA Origami Platform to Organize Snares for Membrane Fusion, J. Am. Chem. Soc. 2016, 138, 4439-47.
20. Czogalla, A.; Kauert, D. J.; Franquelim, H. G.; Uzunova, V.; Zhang, Y.; Seidel, R.; Schwille, P., Amphipathic DNA Origami Nanoparticles to Scaffold and Deform Lipid Membrane Vesicles, Angew. Chem. Int. Ed. 2015, 54, 6501-5.

21. Kocabey, S.; Kempter, S.; List, J.; Xing, Y.; Bae, W.; Schiffels, D.; Shih, W. M.; Simmel, F. C.; Liedl, T., MembraneAssisted Growth of DNA Origami Nanostructure Arrays, ACS Nano 2015, 9, 3530-9.

22. Franquelim, H. G.; Khmelinskaia, A.; Sobczak, J.-P.; Dietz, H.; Schwille, P., Membrane Sculpting by Curved DNA Origami Scaffolds, Nat. Commun. 2018, 9, 811.

23. Grome Michael, W.; Zhang, Z.; Pincet, F.; Lin, C. Vesicle Tubulation with Self-Assembling DNA Nanosprings, Angew. Chem. Int. Ed. 2018, 57, 5330-5334.

24. Yang, Y.; Wang, J.; Shigematsu, H.; Xu, W.; Shih, W. M.; Rothman, J. E.; Lin, C., Self-Assembly of Size-Controlled Liposomes on DNA Nanotemplates, Nat. Chem. 2o16, 8, 476-483.

25. Zhang, Z.; Yang, Y.; Pincet, F.; M, C. L.; Lin, C., Placing and Shaping Liposomes with Reconfigurable DNA Nanocages, Nat. Chem. 2017, 9, 653-659.

26. Conway, J. W.; Madwar, C.; Edwardson, T. G. McLaughlin, C. K.; Fahkoury, J.; Lennox, R. B.; Sleiman, H. F., Dynamic Behavior of DNA Cages Anchored on Spherically Supported Lipid Bilayers, J. Am. Chem. Soc. 2014, 136, 12987-97.

27. Johnson-Buck, A.; Jiang, S. X.; Yan, H.; Walter, N. G., DNA-Cholesterol Barges as Programmable Membrane-Exploring Agents, ACS Nano 2014, 8, 5641-5649.

28. List, J.; Weber, M.; Simmel, F. C., Hydrophobic Actuation of a DNA Origami Bilayer Structure, Angew. Chem. Int. Ed. 2014, 53, 4236-4239.

29. Czogalla, A.; Petrov, E. P.; Kauert, D. J.; Uzunova, V.; Zhang, Y.; Seidel, R.; Schwille, P., Switchable Domain Partitioning and Diffusion of DNA Origami Rods on Membranes, Faraday Discuss. 2013, 161, 31-43; discussion 113-50.

30. Perrault, S. D.; Shih, W. M., Virus-Inspired Membrane Encapsulation of DNA Nanostructures to Achieve in Vivo Stability, ACS Nano 2014, 8, 5132-40.

31. Borjesson, K.; Lundberg, E. P.; Woller, J. G.; Norden, B.; Albinsson, B., Soft-Surface DNA Nanotechnology: DNA Constructs Anchored and Aligned to Lipid Membrane, Angew. Chem. Int. Ed. 2011, 50, 8312-5.

32. Hadorn, M.; Eggenberger Hotz, P., DNA-Mediated SelfAssembly of Artificial Vesicles, PLoS One 2010, 5, e9886.

33. Dave, N.; Liu, J., Programmable Assembly of DNAFunctionalized Liposomes by DNA, ACS Nano 2011, 5, 1304-12.

34. Jakobsen, U.; Simonsen, A. C.; Vogel, S., DNAControlled Assembly of Soft Nanoparticles, J. Am. Chem. Soc. 2008, 130, 10462-10463.

35. Selden, N. S.; Todhunter, M. E.; Jee, N. Y.; Liu, J. S.; Broaders, K. E.; Gartner, Z. J., Chemically Programmed Cell Adhesion with Membrane-Anchored Oligonucleotides, J. Am. Chem. Soc. 2012, 134, 765-8.

36. Koyfman, A. Y.; Braun, G. B.; Reich, N. O., Cell-Targeted Self-Assembled DNA Nanostructures, J. Am. Chem. Soc. 2009, 131, 14237-9.

37. Akbari, E.; Mollica, M. Y.; Lucas, C. R.; Bushman, S. M.; Patton, R. A.; Shahhosseini, M.; Song, J. W.; Castro, C. E., Engineering Cell Surface Function with DNA Origami, Adv. Mater. 2017, 29, 1703632.

38. Göpfrich, K.; Zettl, T.; Meijering, A. E. C.; HernándezAinsa, S.; Kocabey, S.; Liedl, T.; Keyser, U. F., DNA-Tile Structures Induce Ionic Currents through Lipid Membranes, Nano Lett. 2015, 15, 3134-3138.

39. Axelrod, D., Lateral Motion of Membrane Proteins and Biological Function, J. Membr. Biol. 1983, 75, 1-10.

40. Peraro, M. D.; van der Goot, F. G., Pore-Forming Toxins: Ancient, but Never Really out of Fashion, Nature Reviews Microbiology 2015, 14, 77 . 
41. Ros, U.; García-Sáez, A. J., More Than a Pore: The Interplay of Pore-Forming Proteins and Lipid Membranes, $J$. Membr. Biol. 2015, 248, 545-561.

42. Liao, C.; Zhang, Z.; Kale, J.; Andrews, D. W.; Lin, J.; Li, J., Conformational Heterogeneity of Bax Helix 9 Dimer for Apoptotic Pore Formation, Sci. Rep. 2016, 6, 29502.

43. McLaughlin, C. K.; Hamblin, G. D.; Hanni, K. D.; Conway, J. W.; Nayak, M. K.; Carneiro, K. M.; Bazzi, H. S.; Sleiman, H. F., Three-Dimensional Organization of Block Copolymers on "DNA-Minimal" Scaffolds, J. Am. Chem. Soc. 2012, 134, 4280-4286.

44. Edwardson, T. G.; Carneiro, K. M.; McLaughlin, C. K.; Serpell, C. J.; Sleiman, H. F., Site-Specific Positioning of Dendritic Alkyl Chains on DNA Cages Enables Their Geometry-Dependent Self-Assembly, Nat. Chem. 2013, 5, 868-875.

45. Chidchob, P.; Edwardson, T. G.; Serpell, C. J.; Sleiman, H. F., Synergy of Two Assembly Languages in DNA Nanostructures: Self-Assembly of Sequence-Defined Polymers on DNA Cages, J. Am. Chem. Soc. 2016, 138, 4416-4425.

46. Haberland, M. E.; Reynolds, J. A., Self-Association of Cholesterol in Aqueous Solution, Proc. Natl. Acad. Sci. U.S.A. 1973, 70, 2313-6.

47. Khmelinskaia, A.; Franquelim, H. G.; Petrov, E. P.; Schwille, P., Effect of Anchor Positioning on Binding and Diffusion of Elongated 3d DNA Nanostructures on Lipid Membranes, J. Phys. D: Appl. Phys. 2016, 49, 194001.

48. Rassam, P.; Copeland, N. A.; Birkholz, O.; Tóth, C.; Chavent, M.; Duncan, A. L.; Cross, S. J.; Housden, N. G.;
Kaminska, R.; Seger, U.; Quinn, D. M.; Garrod, T. J.; Sansom, M. S. P.; Piehler, J.; Baumann, C. G.; Kleanthous, C., Supramolecular Assemblies Underpin Turnover of Outer Membrane Proteins in Bacteria, Nature 2015, 523, 333.

49. Birkholz, O.; Burns, J. R.; Richter, C. P.; Psathaki, O. E.; Howorka, S.; Piehler, J., Multi-Functional DNA Nanostructures That Puncture and Remodel Lipid Membranes into Hybrid Materials, Nat. Commun. 2018, 9, 1521.

50. Burns, J. R.; Howorka, S., Defined Bilayer Interactions of DNA Nanopores Revealed with a Nuclease-Based Nanoprobe Strategy, ACS Nano 2018, 12, 3263-3271.

51. Ohmann, A.; Li, C.-Y.; Maffeo, C.; Al Nahas, K.; Baumann, K. N.; Göpfrich, K.; Yoo, J.; Keyser, U. F.; Aksimentiev, A., A Synthetic Enzyme Built from DNA Flips 107 Lipids Per Second in Biological Membranes, Nat. Commun. 2018, 9, 2426.

52. Howorka, S., Building Membrane Nanopores, Nat. Nanotechnol. 2017, 12, 619-630.

53. Spruijt, E.; Tusk, S. E.; Bayley, H., DNA Scaffolds Support Stable and Uniform Peptide Nanopores, Nat. Nanotechnol. 2018, 13, 739-745.

54. Garcia-Parajo, M. F.; Cambi, A.; Torreno-Pina, J. A.; Thompson, N.; Jacobson, K., Nanoclustering as a Dominant Feature of Plasma Membrane Organization, J. Cell Sci. 2014, 127, 4995 


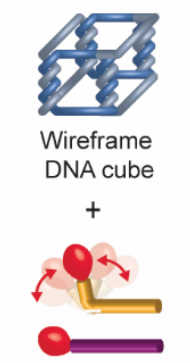

Cholesterol-DNA conjugates
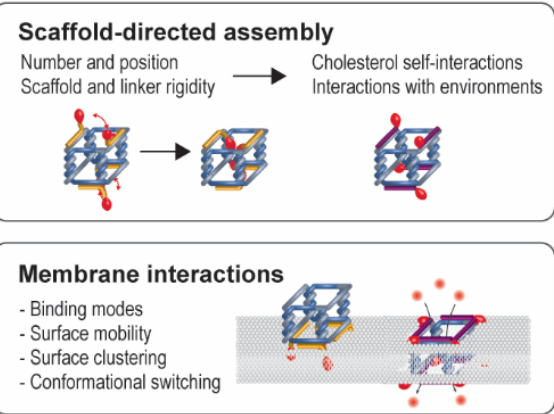